\title{
ARmy: A Study of Multi-User Interaction in Spatially Augmented Games
}

\author{
Andrew Dolce \\ andrewegradientstudios.com
}

\author{
Joshua Nasman \\ nasmajecs.rpi.edu \\ Department of Computer Science \\ Rensselaer Polytechnic Institute
}

\author{
Barbara Cutler \\ cutlerecs.rpi.edu
}

\begin{abstract}
We present ARmy, a two-player military strategy game that uses spatially augmented reality to combine physical tabletop games with the virtual elements and computation characteristic of modern video games. As players move plastic miniatures within a small scale physical environment, the application moderates and augments play by maintaining a 3D representation of the scene, which it uses to validate movement paths and perform automatic line-ofsight calculations for combat. We describe the design and implementation of the ARmy gaming system. Furthermore, we conducted a user study to gauge the effectiveness, intuitiveness, and robustness of the application. We describe the process of this user study, present quantitative data of the study results, and discuss general design principles for the design and implementation of other engaging spatiallyaugmented games.
\end{abstract}

\section{Introduction}

Games are found throughout every day life in a variety of forms, and can be a source of entertainment, a means for education, and even a medium for artistic expression or social commentary. Tangible "tabletop games" include a variety of board, card, and dice games, such as chess, Monopoly, The Settlers of Catan, and Magic: The Gathering. These games use physical pieces to facilitate play. Modern video games provide an experience that is purely virtual, allowing the user to interact with a virtual game world that can include complex simulations and automatic rules enforcement. In contrast, tabletop games hold players responsible for moderating play, which can be difficult and tedious. However, the physically tangible interfaces of tabletop games are often preferable to electronic input devices, which can be challenging and alienating to novice users.

Augmented Reality (AR) has opened up new, exciting and engaging ways for users to view and interact with virtual elements embedded in the real world. We present ARmy (Figure 1), an AR miniature war game played using plas-
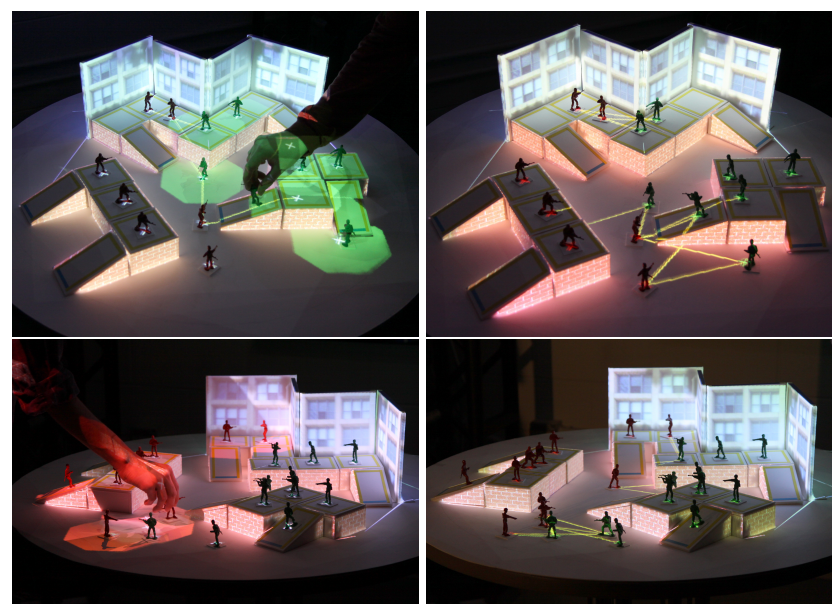

Figure 1. The ARmy game is an example of a spatially augmented reality game that combines physical game objects with virtual elements through projection. The projections decorate simple, white objects with colorful virtual textures, and display important game information regarding legal moves and simulated combat.

tic soldier figurines and physical terrain models in a style similar to Warhammer 40,000 [23]. Multiple projectors are used to directly augment the play surface, displaying useful information about the game state and adding visual detail to the terrain. An overhead camera tracks the movements of the game pieces, and a semi-autonomous game module maintains game state and moderates play. This ensures that the rules are correctly followed and removes the need for players to perform tedious bookkeeping tasks, such as measuring distances or rolling dice to resolve outcomes.

Our contributions presented in this paper:

- Design a robust implementation of a tabletop spatially augmented multiplayer war strategy game.

- A user study demonstrating:

- Improved efficiency of our projector augmented version of the game over the traditional nonaugmented version.

- Accurate, unbiased, and unambiguous enforcement of game rules.

- General guidelines for the creation of other engaging spatially augmented games. 


\section{Related Work}

The technological challenges in augmented reality systems include the creation of effective augmented displays, $3 \mathrm{D}$ registration and tracking, and tangible interaction.

Immersive Display Technology Existing AR systems use a variety of display techniques, which are classified [3] into three main groups: head-mounted, hand-held, and spatially aligned. Head-mounted displays are devices physically worn by the user, e.g., an optical see-though display [29]. Hand-held displays use smart phones or handheld game consoles for video see-through techniques [21, 31, 20]. For example, the ARhrrrr! game is played on a mobile device that overlays graphics onto a physical paper map, and uses tangible, brightly colored candies as input props [7]. Spatially aligned techniques create displays physically aligned with the environment, such as the "Virtual Showcase" display [2]. The ARmy game is an example of Spatially Augmented Reality (SAR) [3], a class of AR systems where the display devices are physically embedded in or projected onto the real-world environment. These environments can surround and immerse the user with largescale projections $[4,25]$. Alternatively, these techniques can be applied at a table-top scale giving complex 3D objects new appearance properties [24]. A theme for projectionbased SAR research is a future in which these technologies become truly ubiquitous [30, 22].

3D Registration and Tracking The virtual game elements and tangible input devices must be aligned and tracked within a common 3D physical environment. One or more fixed cameras placed in the physical environment may be calibrated to a common world coordinate system [33]. The image data from these cameras can then detect, monitor, and track physical objects allowing them to interact with and direct the virtual world in real-time. A large variety of AR applications use fiducial markers to identify objects and determine their position and orientation [10, 5]. However, the robustness and accuracy of the tracking depends on the relative size of these markers. Sufficiently sized tokens may be too large for players to use comfortably and may interfere with the visual quality of virtual elements projected onto them. Unlike see-through AR displays, projector-based SAR systems cannot fully hide the marker with rendered objects.

We have chosen to detect and track the game pieces in ARmy using application-specific colors, shapes, and patterns, similar to [30]. Color-based object recognition is appropriate for augmenting the play of many existing board and card games which have distinctive, brightly-colored elements, allowing detection and tracking without modifying the game pieces. However, color-based techniques can be problematic with inconsistent lighting conditions and background clutter. Alternative methods for interactive

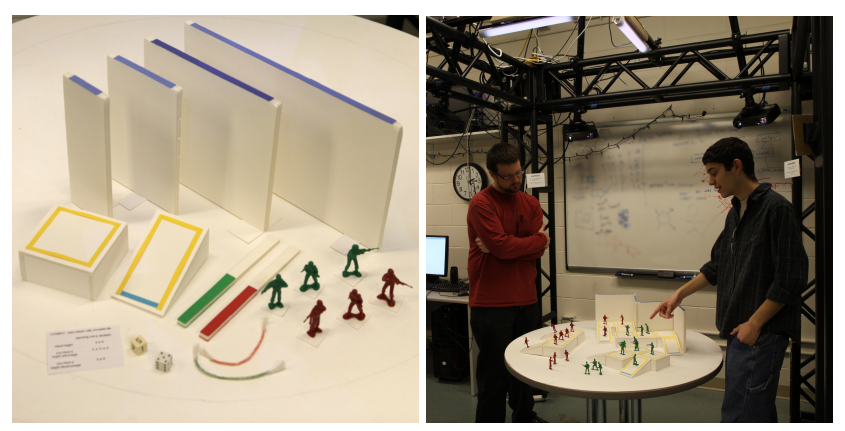

Figure 2. ARmy is played using a set of physical soldier figurines and foam-core terrain objects, including walls, platforms, and ramps. The non-augmented version is played with measuring sticks, string, and dice. The SAR version is equipped with a overhead single camera for object detection and multiple projectors for display.

3D tracking include electromagnetic and mechanical tracking [4], structured light [1, 14], and infrared [15, 11, 18].

Tangible Interaction Tangible user interfaces allow interaction and control of the virtual world by manipulating physical props [6]. Implementation of complex systems with many different types of input and display devices [17] allow users to collaborate in an intuitive manner. The combined virtual and physical world even allows remote users to interact [32] as if they were together physically. Tangible environments allow users to designing a custom space and explore visualizations of the geometry or run simulations that interact with the environment $[8,9]$. IncreTable demonstrates how robots facilitate virtual elements and simulations impact our physical spaces [16].

Tangible interfaces can be applied to education, e.g. teaching billiards [28] and games including tile-based board games [26] and robot battle games [12]. We have designed the ARmy game to mimic existing board games for users familiar with these game dynamics.

\section{ARmy Game Design}

The ARmy application is a military simulation game played between two opponents. Each player controls a set of plastic soldier figurines, referred to as units, which represent their respective armies (Figure 2). On each turn the player moves his units and engages in combat with opposing units to eliminate them from play. The ARmy game is designed as a finite state machine (Figure 3). Using a wireless remote control players send two different signals to the computer game module. The first is an update request that tells the game to capture a new image, detect the current physical game state, and visualize any new information. The second is a continue command indicating that the players have finished with the current stage of play and wish to proceed to the next step. 


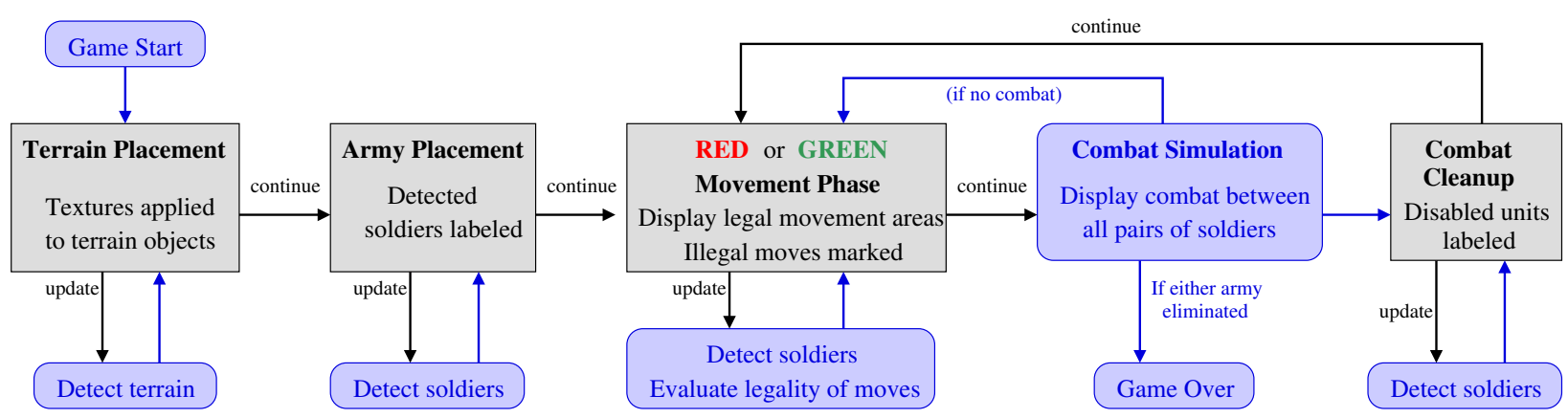

Figure 3. State diagram of the ARmy application. Gray boxes represent stages of play defined by player actions. Blue boxes represent stages performed completely by the game module. Similarly, black arrows represent transitions triggered by the players via the remote control, while blue arrows represent automatic transitions.

Terrain Placement ARmy is played on a varied 3D terrain surface requiring strategic decision making. The ARmy prototype provides three simple terrain object types: 8" vertical walls, elevated platforms (4"x4"x2"), and ramps (3"x5"x2"). Units are not allowed to "jump" or "climb" from low elevation to high elevation or vice versa, but must instead use connected ramps to walk between the two. Controlling elevated terrain and ramps is important to the strategy of the game, as units that hold the higher ground receive a significant advantage in combat. The game begins with a terrain placement step, during which players collaboratively construct the terrain by freely placing provided terrain primitives on the table. Each primitive is constructed from diffuse white foam-core with simple unobtrusive colored markings to facilitate detection. The ARmy application uses SAR projection to apply virtual textures directly onto the physical terrain primitives (Figure 4).

Army Placement Next, players position their respective units (following mutually agreed upon rules), represented by 2" tall red or green plastic soldier figurines. Players may request an update from the game module, which will then detect all positioned figurines, marking each with a projected icon to show that it has been successfully recognized. When all units have been placed to the players' satisfaction, they signal for the game to start.

Movement Phase At the start of a player's movement phase, each of his unit's field of movement is overlaid on the
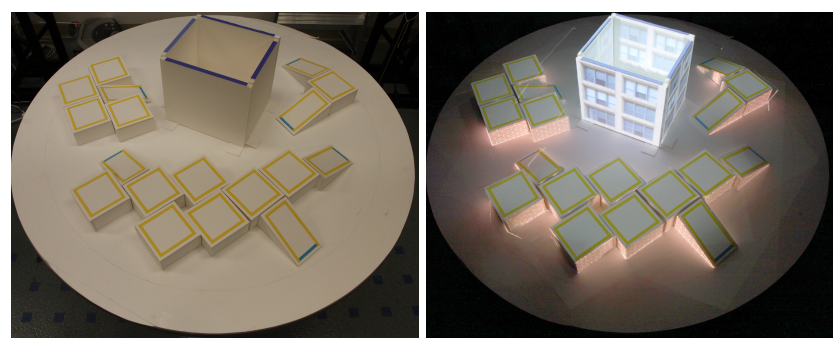

Figure 4. Players freely arrange platform, ramp, and wall terrain objects in the scene, and our system detects and augments these objects with appropriate and interesting textures. terrain as a colored region (Figure 5). Each unit's movement is constrained to a maximum distance of 4" and must conform to the terrain rules described earlier. The player may move any number of his units, and each unit may move anywhere within its corresponding highlighted region. At any point, the player may request an update of the visualization from the game module, which will then augment the overlaid display with a visualization of the detected movements (Figure 6). In the event that a given move is invalid, this information is reflected in the overlay, allowing the player to correct the error.

In the non-augmented version of this type of game, players measure distances using a ruler or measuring tape. Augmentation is helpful in automating tedious tasks and moderating subjective calls that could lead to disagreements between players about the legality of specific moves. In the augmented version, the system clearly and precisely indicates each unit's field of movement, and marks illegal moves. Additionally, the system tracks and displays each unit's original position, thereby avoiding a potential source of confusion for complex multi-unit battle scenarios.

Combat Simulation Each movement phase is followed by a combat simulation round, during which all units automatically attack non-occluded targets in a range of 8 ". This is similar to combat in a typical real-time strategy (RTS) video game, in which smart units exhibit autonomy in their
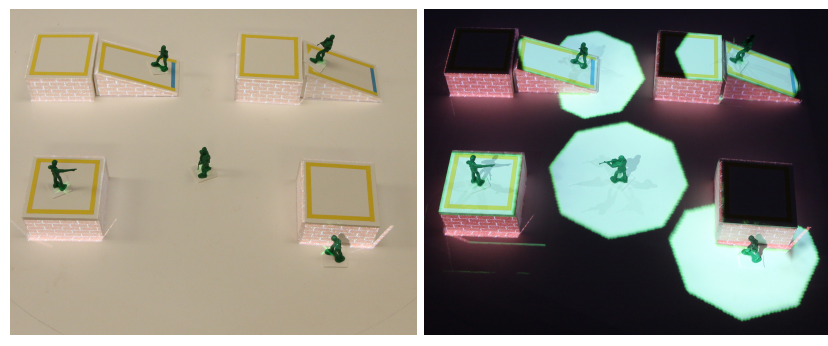

Figure 5. During the movement phase, each unit's field of movement is illuminated. Note that the visualization conforms to movement rules across terrain boundaries, which require the use of ramp objects in order to climb up or down elevated platform. 

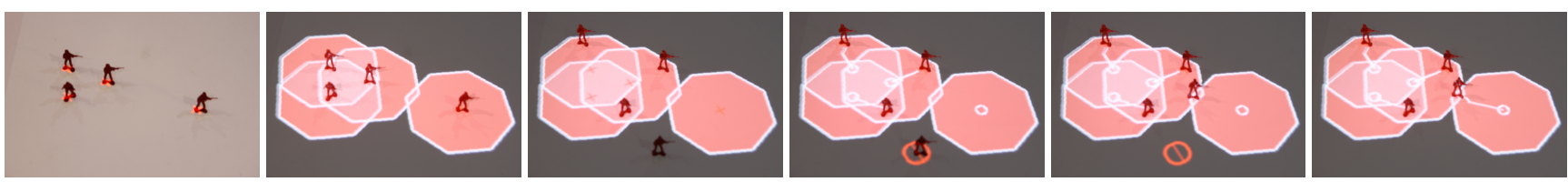

Figure 6. During each movement phase the player may move all of their units simultaneously. The system matches each unit's current position (marked with a " $\times$ " icon) to its previous position (marked with a "o" icon) using the Hungarian Method (drawn as a white path). If no legal move is found the unit is marked appropriately with a "not" symbol. The player must then correct the error before proceeding.

actions. Colored combat line visualizations are overlaid on the ground textures, connecting opposing units that are able to attack each other (Figure 7). This visualization provides important feedback to help players plan moves strategically. During a subsequent combat round, the game module iterates through all connected pairs and simulates an individual contest, which may result in one or both units being eliminated. In our game, a unit on equal or lower ground than an opposing unit has a $1 / 3$ chance of disabling the opposing unit. A unit on higher ground than the opponent has a $2 / 3$ chance of disabling the opponent. In a non-augmented game, this is determined by rolling a 6 -sided die.

\section{ARmy Implementation Details}

The ARmy application leverages an existing generalpurpose multi-projector SAR system [27] that facilitates a variety of tangible education and gaming applications.

Object Detection During acquisition, a single color image from the calibrated overhead camera (Figure 8) is processed by our vision module, which uses thresholding techniques to identify connected image components with predominant color attributes matching a set of identifiable colors. The vision module classifies each component as one of the known game object primitives. During the terrain placement phase, the system detects the initial configuration of terrain objects, which are encoded with the colors blue, yellow, and cyan. During the army placement and
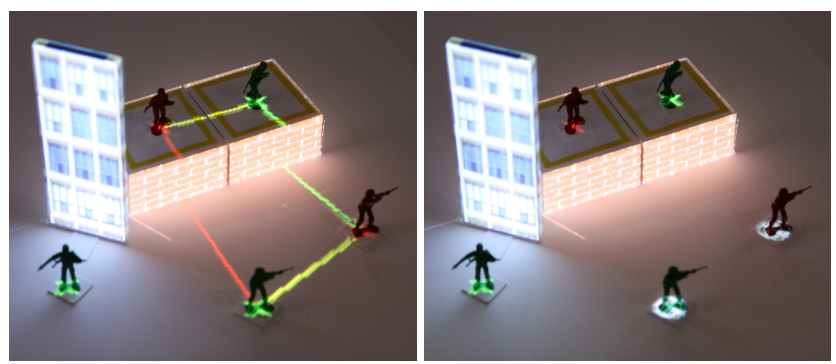

Figure 7. Brightly colored combat lines indicate which units are within firing range and have a line of sight to an opposing unit. Yellow lines indicate combat between units on equal height. A red line indicates a height advantage for the red player and similarly a green line indicates a height advantage for the green player. Note that the leftmost green unit's line of sight to one red unit is blocked by the wall and the other red unit is more than 8 inches away. After the combat round, two units are marked for removal (" $\otimes$ " icon). movement phases, the vision module focuses on appropriately sized red and green colored components that indicate the location of a soldier figurine. Overall, our color-based detection method successfully and robustly recognizes a variety of objects without the need for large fiducial markers or embedded sensors, allowing us to easily detect and track a sizable collection of simple game objects.

Modeling the Game World The terrain geometry is processed to remove unwanted discontinuities, such as small gaps between adjacent platforms and ramps that would undesirably block the movement of units. The resulting heightfield (Figure 8e) is used to construct a valid movement graph for calculation of travel distances as units move through the scene. Two adjacent pixels are connected only if the difference in height is less than a prescribed elevation difference threshold.

Tracking Soldier Movement To determine each unit's valid movement options, the game module performs a breadth-first search of the movement graph limited by the unit's maximum movement distance. The player is allowed to simultaneously move any number of his units, requesting a new scene acquisition only when he would like to see an updated display or has decided on a final configuration. Unfortunately, our current tracking method does not uniquely identify each unit (it only distinguishes red versus green) and we did not want to limit players to moving a single unit between updates, as this would disrupt play. Thus, the game module must deduce the likely correspondence between the units' current and previous locations using the Kuhn-Munkres algorithm [13, 19]. Given the $m$ 2D locations of a player's units before an update, and a set of $n$ locations representing the newly detected positions, we construct a $m \times n$ matrix $A$, such that each coefficient $A_{i j}$ is equal to the assignment cost of matching the $i^{\text {th }}$ unit of the old configuration to the $j^{\text {th }}$ unit of the new configuration. The algorithm solves for the minimum cost correspondence between the locations.

Line of Sight Calculation We use the 2.5D heightfield representation of the game world to perform automatic visibility tests between opposing units.

Visual Augmentation The positions and orientations of detected terrain objects are used to create a virtual 3D mesh representation of the scene. The ARmy application generates textures for each of the surfaces in the mesh and the 

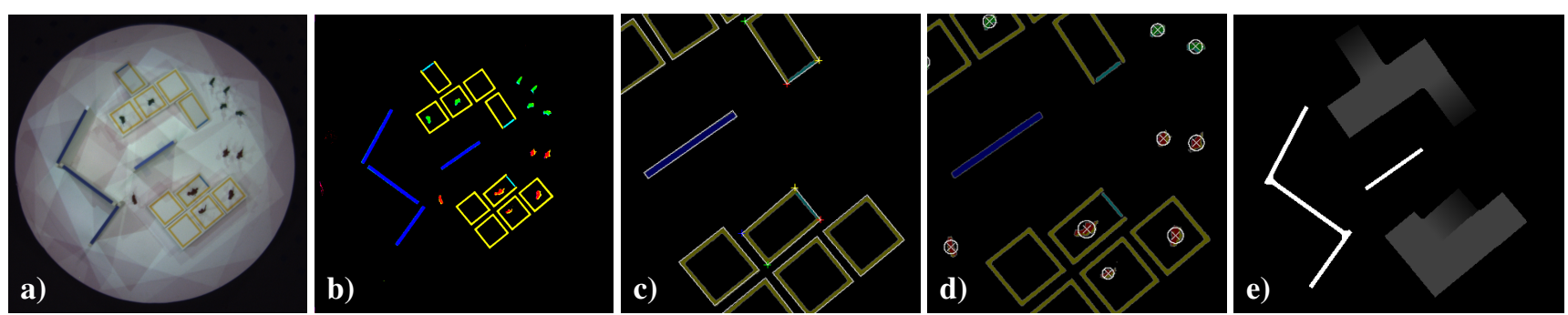

Figure 8. a) Raw camera images of the scene show variable lighting due to shadows and regions of projector overlap. The vision component ignores these artifacts by considering b) only pixels with highly saturated color properties, which it groups into connected components. These components are classified as c) quadrilateral terrain regions or d) soldier figurines based on size, color, and shape. e) The final heightfield after dilation and erosion to fill in unwanted discontinuities.

SAR system coordinates the display of this information into the scene using the calibrated projectors.

\section{ARmy Design and Interaction User Study}

The first goal of our user study was to determine if interaction with the system was natural and intuitive, and to judge the learning curve for users familiar with physical board games and computers but new to spatially augmented reality. A companion goal was to assess the stability and robustness of our SAR system in a full game scenario with non-developers. Most importantly, we wanted to solicit feedback on the visualization elements and overall gameplay. To provide a baseline for comparison, all study participants played both a traditional, non-augmented version of the ARmy game as well as the projector augmented game. We hypothesized that the augmented version would be less tedious, less ambiguous or contentious, and that movement and combat would be more efficient. Altogether, this would allow users to play more rounds of the game and explore and evaluate more complex gaming strategies. We also hypothesized that users would find the augmented reality technology more engaging and immersive than the traditional version of the game.

Goals for User Study Design We designed the study as a direct comparison of the same basic game played two different ways: using traditional non-augmented technology (rulers \& dice), and using the projector augmentation. We held constant the game rules, including the turn sequence, movement restrictions, combat sight lines, and combat probabilities. After an introduction to the SAR system and a brief description of the games rules $(\sim 10 \mathrm{~min}-$ utes), the participants played the game three times. The preliminary game was for practice ( $\sim 15$ minutes) in which the participants used both the traditional mechanisms of rulers and dice and the projected visualizations of movement areas and combat circles. In the practice round each player started with 5 units and we encouraged the participants to set up near their opponent to ensure they gained experience with the combat rules. Most participants played 1 or 2 full cycles of gameplay (movement for each player and joint combat after each movement phase). Next, the participants played two full games, one with and one without augmentation, in a randomly-selected order. For each of the full games, players started with 12 units each and played for a maximum of 20 minutes. Participants were specifically not allowed to use rulers and dice when playing the augmented game. Similarly, all projector visualization and texturing was disabled for the non-augmented version. The supplementary video shows sample footage of both the augmented and non-augmented versions of the movement and battle phases of the game. The script (read aloud to participants) for the user study is included as supplementary material.

Background of Study Participants We believe it is important to find participants who enjoy playing games and have a sense of competitiveness, strategy, and intellectual curiosity when doing so. Thus, we recruited artists and computer scientists from the Games and Simulation Arts and Sciences undergraduate major. We had a range of participants from freshmen through graduate students. In total, 26 users participated in the initial pilot study ( 3 females, 5 males) or the main study ( 6 females, 12 males). We summarize the background for the participants in the main study: 13 of the 18 participants have $0.5-5$ years formal education in game studies. 12 of the participants have at least 3 years formal education in computer science. 11 of the participants have at least 1 year of formal art education, 7 have at least 4 years art education. All users have at least 3 years experience playing computer games, 14 had more than 10 years experience. All users have experience playing board games, 15 of them have more than 10 years experience. Half of the users had prior experience (0.5-3 years) with table top games similar to Warhammer 40,000.

Written Questionnaire At the end of play, each participant filled out a written questionnaire (supplementary material) directly comparing the augmented and non-augmented versions of the game for several important gameplay characteristics (Table 1). The average rating for all 18 study participants for each version of the game is provided. We also separately average the ratings of the users who played that 


\begin{tabular}{|c|c|c|c|c|c|}
\hline \multirow{2}{*}{$\frac{\text { avg. rating }}{\text { acc. distance }}$} & \multicolumn{2}{|c|}{$\begin{array}{c}\text { non-augmented } \\
\text { all(18) } 2^{\text {nd }} \text { only }(10)\end{array}$} & \multicolumn{2}{|c|}{$\begin{array}{c}\text { augmented } \\
\text { all(18) } 2^{n d} \text { only(8) }\end{array}$} & \\
\hline & 3.7 & 4.0 & 4.5 & 4.1 & 0.8 \\
\hline acc. sight lines & 3.8 & 4.0 & 4.8 & 4.6 & 0.9 \\
\hline acc. rules & 3.8 & 3.9 & 4.6 & 4.4 & 0.8 \\
\hline subjectivity & 4.1 & 3.9 & 4.6 & 4.6 & 0 \\
\hline interest & 3.5 & 3.9 & 4.6 & 4.6 & 1 \\
\hline
\end{tabular}

Table 1. Participant's rating of the accuracy of distance calculations, line-of-sight judgments, and implementation of rules, their assessment of the subjectivity of rule enforcement, and their overall interest while playing the game. Each is scored on a scale of 1 to 5 , with 5 being the positive attribute quality.

version of the game as their second playthrough (when they were more familiar with the game mechanics, rules, and strategy). Overall, the ratings indicate that participants were interested in playing both games, thought that the different versions accurately represented the game mechanics, found enforcement of rules was not too subjective, felt that the game was fair, and rarely disagreed with each other or with the computer. Using Single Factor ANOVA, users rated the augmented version more positively than the non-augmented with a $p$ value of .005 or lower in every case except subjectivity. Users rated subjectivity higher with a $p$ value of .08 .

Timing Results A video camera recorded each experiment allowing us to measure the time for terrain setup, initial army placement, average time for each player's movement phase (red or green), average time for a combat round, and average time for a battle (when two units face off, requiring each player to roll once). We also counted the number of rounds (red move/combat/green move/combat) per game, and the number of battles per game (Table 2). This data allows us to compare the efficiency of play with and without augmentation. We present the data averaged over all experiments and a separate average of the games played as the second full playthrough. Note: Due to video errors, a few of the game recordings are incomplete and omitted from these averages.

Game setup is slower with the augmented system for both terrain layout and unit placement, due to a number of minor factors: triggering the remote, waiting for the visualization to refresh, and reminding players to remove unnecessary materials from the table and step out of the camera's field of view. Similarly, movement phases are slower in the augmented version because moves are validated by the system and the extra time required to correct illegal moves. With SAR system overhead optimization we believe these differences can be greatly reduced or eliminated. In particular, we believe these improvements combined with user familiarity with the movement region visualization will allow movement phases to be faster in the augmented version than in the non-augmented version.

Not-surprisingly, the augmented system's main effi-

\begin{tabular}{l|cc|cc} 
& \multicolumn{2}{|c|}{ non-augmented } & \multicolumn{2}{c}{ augmented } \\
averages & all(9) & $2^{\text {nd }}$ only(3) & all(9) & $2^{\text {nd }}$ only(5) \\
\hline terrain setup & $1: 16$ & $1: 13$ & $2: 46$ & $2: 07$ \\
army placement & $2: 52$ & $2: 38$ & $3: 03$ & $3: 11$ \\
movement phase & $0: 42$ & $0: 36$ & $0: 52$ & $0: 47$ \\
combat phase & $1: 33$ & $2: 13$ & $0: 22$ & $0: 18$ \\
single battle & $0: 16$ & $0: 21$ & $0: 04$ & $0: 03$ \\
\hline \# rounds per game & 2.3 & 2.0 & 3.9 & 4.1 \\
\# battles per game & 26.1 & 24.5 & 36.6 & 41.0
\end{tabular}

Table 2. The average timing data (minutes:seconds) for various stages of play are summarized for both non-augmented (traditional) and SAR augmented experiments.

ciency improvement is gained in the combat simulation phase ( $\sim 4 \mathrm{X}$ faster). The simulation of each battle is visualized one-at-a-time for the players $(\sim 1$ second per virtual "die" roll). Note that the combat phase also includes removal of disabled units. The greatest efficiency improvement is in calculating which units have line of sight and are in range, and most importantly, in keeping track of which battles have occurred and correctly accounting for all combinations of opposing units when they are densely clustered.

As we hypothesized, overall the augmented version allows players to complete more cycles of play before time is called (60\% more), and similarly, more total battles are fought ( $40 \%$ more) in the augmented version.

Quantitative Analysis of Unit Movements Next, we analyzed the unit movements in augmented games. We were interested in quantifying the fraction of unit movements that were close to the maximum 4" distance. We also examined all cases in which a unit was moved slightly beyond this maximum distance and marked as an illegal move. Table 3 summarizes the data for a total of 678 unit movements. $60 \%$ of unit movements were "big moves", reaching more than $75 \%$ of the maximum distance and $25 \%$ of the moves pushed within a pixel of the 4" movement radius. We assume that for most of these moves the user was strategically interested in maximizing unit movement. However, they may have shied away from making a full 4" movement to avoid having that move marked as illegal. 9 out of 14 pairings saw at least one borderline unit movement marked as illegal during either the practice round or the full game. When a movement is marked illegal, it causes an interruption in game flow, requiring correction of the error. In most cases, the player over-corrected for the error, backing up the soldier to approximately $75 \%$ of the full move. In a few cases, the player was confused about which unit moved too far, and conservatively adjusted several other legal moves.

Only twice during our study did the system catch flagrantly illegal moves, and neither case was malicious. In one instance the illegal move came in the confusion immediately after a detection error. We found that detection errors during play were rare, proving that our prototype sys- 


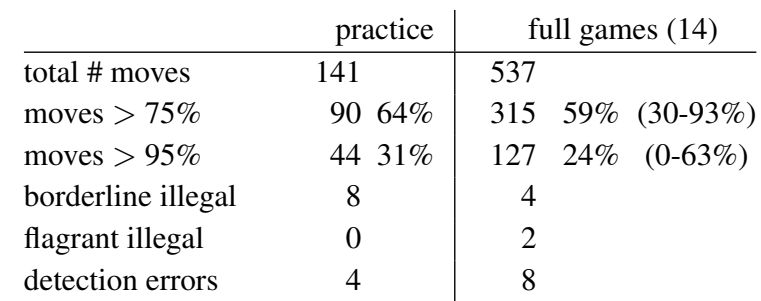

Table 3. A summary of the individual ARmy unit movement data for the augmented gameplay during both practice and full games.

tem is robust, engaging, and thoroughly playable. Detection errors occurred when units were placed too close together and detected as a single unit, when a unit hidden behind a wall was not visible to the camera, and a few rare color thresholding errors. Other detection errors were related to game rule ambiguities; for example, straddling a unit between platforms that touch only at the corner or balancing units on the very edge of a platform.

Verbal and Written Feedback We encouraged participants to ask questions and offer feedback throughout the study. Each participant also answered several short written answer questions. A summary of the participant comments:

Positive Aspects of Traditional Game: "It was a little more hands on" and "player decisions were more fluid", which "keeps players actively involved in game play". Tactile control allowed participants to "know exact outcome of dice" and they "felt responsible for the outcome of the dice". Users appreciated the "slight bending of rules for more realistic and entertaining game play".

Negative Aspects of Traditional Game: "Much slower combat (actually rolling the dice each time and making sure every combination of soldiers is accounted for)" and "when many attacks [happened] at the same time, it made the game stop and not be fun". "In a dense soldier cluster there were so many attacks made that we probably lost count at some point and either attacked too many times or too few". Participants experienced "occasional confusion (even about whose turn it was)" and "While we made decisions, they were not necessarily the correct ones in terms of the rules".

Positive Aspects of Augmented Game: Participants found that the "visualizations were easy to understand", it was "easy to see how far you can move", and it was "never unclear about whose turn it was or what could be done". Most participants "trust [the] computer" and the games had "no disagreement between players since there was an ultimate referee". The use of projective texture was "more visually stimulating" and the animations of "the arrows for combat were amusing to watch". Participants specifically commented that faster, more efficient play made it "much easier to establish movement strategy" because "it allowed you to focus on the game play and not the math behind it".

Negative Aspects of Augmented Game: "Turns were slow" while "waiting for recalculation" and "moving pieces slightly out of range made it take longer sometimes". Occasional system glitches and the "restriction in terrain placement because of projection" were negatives. "Blind spots forced us to simplify our first terrain design a bit." Explanation of game results was sometimes unclear: "Don't know the reason the soldier was disabled". "Took the player engagement away a bit. Watched action happen rather than rolling the dice. Takes away your feeling of involvement."

\section{Discussion and Conclusions}

Using affordable, off-the-shelf hardware we created an immersive SAR game experience that 15 out of 18 participants preferred over the non-augmented version. Two preferred the non-augmented version and one person called it a tie. We believe that many other physical board and card games could similarly be positively augmented and improved by such a setup. Depending on the system budget and physical game environment and components, this infrastructure could be simplified to use a single projector or expanded to use multiple cameras or alternate tracking technology (Section 2). This system is practical for installation in home "game room" environments.

Augmented gaming systems should facilitate and encourage users to make optimal decisions; for example, in ARmy this often means making a full 4" distance movement. Unfortunately, we found that some users were reluctant to make maximal movements because the delay in correcting an overmove interrupted game play. Instead of forcing users to nudge pieces back, the system could automatically recognize these slight over-movements, and clamp the internal representation of the unit's position to the maximum distance. We recommend that similar tolerances be incorporated when possible in all AR based games.

Users were excited by the system ease-of-use and efficiency of the battle rounds. Game setup occasionally took longer when technical problems occurred with the prototype SAR system. These system issues must be resolved prior to commercialization of this type of technology. It is important that users feel engaged and in control while playing SAR games. Even though the combat rounds were more efficient in the augmented version, some users wished they had more control over the automated battle sequences and dice rolls. Future studies are needed to determine the optimal timing of user interactions and automated system computation. More visualization of the simulated die rolls and the ability to specify individual attacks instead of fully automated combat may be beneficial. Some users noted that the game state could change significantly between turns. It was not uncommon for one half of an army to be eliminated in a single combat round. More complexity in the game rules, for example "hit points" for each unit would make the game more interesting and require more strategy.

By adapting our SAR framework to facilitate simulta- 
neous acquisition and display, the gaming module could more naturally react to users without the need for turn-based update requests. Gesture-based or verbal speech controls, could also be beneficial. Gameplay could be made more engaging by improvements to the detection sequence and in finding ways for users to interact during combat. Finally, play could be enhanced by displaying state information directly onto the units to remove clutter, and by adding audio elements to increase the immersive feel of the game.

Overall, the ARmy application is a fully functional prototype that demonstrates the key benefits of SAR for tangible gaming. The results of our user study indicate a general participant preference for the augmented version of our game prototype, and feedback has shown a positive opinion about SAR technology in the context of tabletop games.

\section{References}

[1] M. Ashdown, M. Flagg, R. Sukthankar, and J. M. Rehg. A Flexible Projector-Camera System for Multi-Planar Displays. In Proc. of the 2004 IEEE Computer Society Conf. on Computer Vision and Pattern Recognition (CVPR), pages 165-172, 2004. 2

[2] O. Bimber, B. Fröhlich, D. Schmalstieg, and L. M. Encarnação. The Virtual Showcase. In ACM SIGGRAPH 2005 Courses, 2005. 2

[3] O. Bimber and R. Raskar. Spatial Augmented Reality: Merging Real and Virtual Worlds. A K Peters LTD, 2005. 2

[4] C. Cruz-Neira, D. J. Sandin, and T. A. DeFanti. Surround-Screen Projection-Based Virtual Reality: The Design and Implementation of the CAVE. In Proc. of the 20th Ann. Conf. on Computer Graphics and Interactive Techniques (SIGGRAPH), pages 135-142, 1993. 2

[5] M. Fiala. ARTag, a Fiducial Marker System Using Digital Techniques. In Proc. of the 2005 IEEE Computer Society Conf. on Computer Vision and Pattern Recognition (CVPR), volume 2, pages 590596. IEEE Computer Society, 2005. 2

[6] G. W. Fitzmaurice, H. Ishii, and W. A. S. Buxton. Bricks: laying the foundations for graspable user interfaces. In Proc. of the SIGCHI conference on Human factors in computing systems, CHI '95, pages 442-449. ACM Press/Addison-Wesley Publishing Co., 1995. 2

[7] Georgia Institute of Technology Augmented Environments Lab. ARhrrrr! http://www.augmentedenvironments.org/lab/research/ handheld-ar/arhrrrr/ (Last Accessed: 4/19/11). 2

[8] H. Ishii, C. Ratti, B. Piper, Y. Wang, A. Biderman, and E. BenJoseph. Bringing clay and sand into digital design continuous tangible user interfaces. BT Technol J, 22(4):287-299, Oct. 2004. 2

[9] B. R. Jones, R. Sodhi, R. H. Campbell, G. Garnett, and B. P. Bailey. Build your world and play in it: Interacting with surface particles on complex objects. In ISMAR, pages 165-174. IEEE, 2010. 2

[10] H. Kato and M. Billinghurst. Marker Tracking and HMD Calibration for a Video-Based Augmented Reality Conferencing System. In Proc. of the 2nd IEEE and ACM Int. Workshop on Augmented Reality, page 85. IEEE Computer Society, 1999. 2

[11] Kinect. Microsoft, November 2010. http://www.xbox.com/ enUS/kinect (Last Accessed: 4/20/11). 2

[12] M. Kojima, M. Sugimoto, A. Nakamura, M. Tomita, M. Inami, and H. Nii. Augmented Coliseum: An Augmented Game Environment with Small Vehicles. In Proc. of the First IEEE Int. Workshop on Horizontal Interactive Human-Computer Systems, TABLETOP '06, pages 3-8. IEEE Computer Society, 2006. 2

[13] H. W. Kuhn. The Hungarian Method for the Assignment Problem. Naval Research Logistics Quarterly, 2:83-97, 1955. 4

[14] J. C. Lee, P. H. Dietz, D. Maynes-Aminzade, R. Raskar, and S. E. Hudson. Automatic Projector Calibration with Embedded Light Sen- sors. In Proc. of the 17th Ann. ACM Sym. on User Interface Software and Technology, pages 123-126, 2004. 2

[15] J. C. Lee, S. E. Hudson, and P. H. Dietz. Hybrid Infrared and Visible Light Projection for Location Tracking. In Proc. of the 20th Ann. ACM Sym. on User Interface Software and Technology, pages 5760, 2007. 2

[16] J. Leitner, M. Haller, K. Yun, W. Woo, M. Sugimoto, and M. Inami. Incretable, a mixed reality tabletop game experience. In Proc. of the 2008 Int. Conference on Advances in Computer Entertainment Technology, ACE '08, pages 9-16. ACM, 2008. 2

[17] A. MacWilliams, C. Sandor, M. Wagner, M. Bauer, G. Klinker, and B. Bruegge. Herding sheep: Live system development for distributed augmented reality. In Proc. of the 2nd IEEE/ACM Int. Sym. on Mixed and Augmented Reality, ISMAR '03, pages 123-, 2003. 2

[18] F. Maimone. Reducing Interference Between Multiple Structured Light Depth Sensors Using Motion. IEEE VR '12, 2012. 2

[19] J. Munkres. Algorithms for the Assignment and Transportation Problems. J. Soc. Ind. Appl. Math., 5(1):32-38, 1957. 4

[20] Nintendo. 3DS, 2011. http://www.nintendo.com/3ds (Last Accessed: 4/19/11). 2

[21] W. Pasman and C. Woodward. Implementation of an Augmented Reality System on a PDA. In Proc. of the 2nd IEEE/ACM Int. Sym. on Mixed and Augmented Reality (ISMAR), pages 276-. IEEE Computer Society, 2003. 2

[22] C. S. Pinhanez. The Everywhere Displays Projector: A Device to Create Ubiquitous Graphical Interfaces. In Proc. of the 3rd Int. Conf. on Ubiquitous Computing, pages 315-331. Springer-Verlag, 2001. 2

[23] R. Priestley and A. Chambers. Warhammer 40,000. Games Workshop, 1987. http://www.games-workshop.com/ gws/home.jsp (Last Accessed: 4/19/11). 1

[24] R. Raskar, K. Low, and G. Welch. Shader Lamps: Animating Real Objects with Image-Based Illumination. Technical report, University of North Carolina at Chapel Hill, 2000. 2

[25] R. Raskar, G. Welch, M. Cutts, A. Lake, L. Stesin, and H. Fuchs. The Office of the Future: A Unified Approach to Image-Based Modeling and Spatially Immersive Displays. In Proc. of the 25th Ann. Conf. on Computer Graphics and Interactive Techniques (SIGGRAPH), pages 179-188. ACM, 1998. 2

[26] M. Rooke and R. Vertegaal. Physics on display: tangible graphics on hexagonal bezel-less screens. In Proc. of the 4th Int. Conf. on Tangible, Embedded, and Embodied Interaction, TEI '10, pages 233-236. ACM, 2010. 2

[27] Y. Sheng, T. C. Yapo, C. Young, and B. Cutler. Virtual Heliodon: Spatially Augmented Reality for Architectural Daylighting Design. In IEEE Virtual Reality Conf. (VR), pages 63-70. IEEE Computer Society, 2009. 4

[28] A. Suganuma, Y. Ogata, A. Shimada, D. Arita, and R.-i. Taniguchi. Billiard instruction system for beginners with a projector-camera system. In Proc. of the 2008 Int. Conference on Advances in Computer Entertainment Technology, ACE '08, pages 3-8. ACM, 2008. 2

[29] I. E. Sutherland. A Head-Mounted Three Dimensional Display. In Proc. of the Fall Joint Computer Conf., Part I (AFIPS), pages 757764. ACM, December 1968. 2

[30] J. Underkoffler, B. Ullmer, and H. Ishii. Emancipated Pixels: RealWorld Graphics in the Luminous Room. In Proc. of the 26th Ann. Conf. on Computer Graphics and Interactive Techniques (SIGGRAPH), pages 385-392. ACM, 1999. 2

[31] D. Wagner and D. Schmalstieg. First Steps Towards Handheld Augmented Reality. In Proc. of the 7th IEEE Int. Sym. on Wearable Computers (ISWC), pages 127-. IEEE Computer Society, 2003. 2

[32] A. D. Wilson and D. C. Robbins. PlayTogether: Playing Games across Multiple Interactive Tabletops IUI'07 Workshop on Tangible Play. 2

[33] Z. Zhang. A Flexible New Technique for Camera Calibration. IEEE Trans. on Pattern Analysis and Machine Intelligence, 22(11):1330 1334, November 2000. 2 